\title{
A Content-based Image Retrieval System Based On Convex Hull Geometry
}

\author{
Santhosh P. Mathew ${ }^{1}$, Valentina E. Balas ${ }^{2}$, Zachariah K. P. ${ }^{1}$ \\ ${ }^{1}$ Department of Computer Science, Saintgits College of Engineering, Kerala, \\ India; E-mail: santhosh.mathew@saintgits.org, zacharia.kp@ saintgits.org \\ ${ }^{2}$ Department of Automatics and Applied Software, Aurel Vlaicu University of \\ Arad, Romania; E-mail: valentina.balas@uav.ro
}

\begin{abstract}
Developments in data storage technologies and image acquisition methods have led to the assemblage of large data banks. Management of these large chunks of data in an efficient manner is a challenge. Content-based Image Retrieval (CBIR) has emerged as a solution to tackle this problem. CBIR extracts images that match the query image from large image databases, based on the content. In this paper, a novel approach of comparing the convex hull geometry of the query image to that of the database image in terms of a relative metric which is denoted as the Convex Hull Area Ratio (CHAR) is used. The metric CHAR is the ratio of the area of the intersection of the two convex hulls to the area of their union. Convex hull shape polygon is extracted from the database images and the coordinate values are stored in the feature library. When a query image is given, the convex hull values are extracted in the same fashion. Ratio of the intersected area to union area of the two convex hulls (CHAR) are found and stored in an array. Subsequently, similarity measurement is performed and the maximum value of the CHAR indicates the closest match. Thus, the database images that are relevant to the given query image are retrieved. Scale and translational invariance have been preserved by a suitable co-ordinate transformation. The proposed CBIR technique is evaluated by querying different images and the retrieval efficiency is evaluated by determining precision-recall values for the retrieval results.
\end{abstract}

Keywords: Image Retrieval; Shape Signature; Image Segmentation; Edge detection; Convex hull; Area ratio

\section{Introduction}

Content-based Image Retrieval system is an information processing system that makes use of the image content in the retrieval process. Design and development of an automated retrieval system is quite difficult, considering the complex objects and information present in the image. An image retrieval system can be defined as a computer system for browsing, searching and retrieving images from a 
large database of digital images. Some of the most important characteristics that are used to extract information from the images are Color, Shape and Texture.

Color histograms are commonly used in Content-based image retrieval. Though Color and texture contain important information, it is possible that two images with similar color histograms may actually represent very different images. Hence, shape describing features play a vital role in effectively retrieving images by making use of a CBIR system. Though much research is going on in shape based representation and retrieval techniques, there is no direct solution as to which kind of shape features are to be considered for best performance [18].

In this paper, a novel approach of comparing the two convex hulls of the prominent edges in the query image to that of the database image by computing the Convex Hull Area Ratio (CHAR) is made use of. The proposed CBIR system effectively retrieves the images relevant to the query image when compared to the other CBIR system. The remaining part of the paper is organized as follows: Section 2 discusses some of the related works. Section 3 briefs the Content-based image retrieval with proposed shape signature extraction, feature calculation and image retrieval process. Results and analysis of the proposed technique are discussed in Section 4. Finally, concluding remarks are provided at the end.

\section{Background of the Research Work}

Tools that effectively browse, search and retrieve images are needed by users from different domains. Remote sensing, Fashion, Crime prevention, Publishing, Medicine, Architecture, etc. are some of them. Image retrieval has been in the thick of research for the past many decades. Database management and Computer vision are the two major research groups who have been contributing to this field. These groups study image retrieval from different perspectives. Text based approach is adopted by the former, while the latter follows content-based approach. High level features like keywords and text descriptors are normally used by humans to interpret images and measure their similarity. But the low level features automatically extracted using computer vision methods are normally of low level in nature [1] [2]. Early techniques of image retrieval were based on the manual textual annotation, a difficult and laborious task. Texts alone are not sufficient because the interpretation of what we see is hard to characterize by mere words. This led to the contents in an image, like color, shape, and texture, gaining more importance and in turn in the birth of CBIR [3].

Shape is considered to be one of the most important low level image features in content-based image retrieval. Region-based and Contour-based are the two common approaches used by shape-based systems [4]. Region-based systems normally use Geometrical moments, Zernike moments, Legendre moments and such moment descriptors [5] [6]. Contour-based systems use the boundary of the 
objects. We usually get better results for images that are distinguishable by their contours. Literature discusses various shape representation and retrieval techniques. FD (Fourier Descriptors), PA (Polygonal Approximation), IM (Invariant Moments), CSS (Curvature Scale Space) etc. are some of them [7]. Shape is a major component used for describing digital image along with other features such as color and texture. Contour-based shape descriptors, such as Fourier descriptors, are not appropriate for describing shapes consisting of several disjoint regions. Region based shape descriptors, such as moments, are used when shapes have complex boundaries [8].

Experiments on CBIR systems show that, on many occasions, low level contents fail to describe the high level semantic concepts. Ying Liu et al. [9] provided a comprehensive survey of the recent technical achievements in high-level semantic based image retrieval. Their research covered different aspects, including low level image feature extraction, similarity measurement, and deriving high level semantic features.

Manual textual annotation of images was made use for the earlier image retrieval systems. This was a very difficult and time consuming task. It is very difficult to describe what we see and interpret purely using text. This led to the contents in an image like color, shape, and texture gaining more importance. Amit Jain et al. [3] proposed an algorithm for retrieving images with respect to a database consisting of engineering/computer aided design (CAD) models. Retrieval of objects has been done using a similarity measure that combines shape and the depth information. They combined the shape obtained from the contour with the 3D embedding of the depth information at each point on the contour, to identify a feature set. Trademark Image Retrieval (TIR) was developed to check and prevent the repetition of the large number of trade mark images that are stored in the trademark registration system. Chia -Hung Wei et al. [6] derived a Content-based trademark retrieval system with a set of feature descriptors that could represent global shapes and local features of the trademarks.

Many retrieval systems use norm based distances on the extracted feature set as a similarity function. H. B. Kekre et al. [10] suggested that complex Walsh transform sectors of the images could be used to generate the feature vectors for the purpose of image search and retrieval. Xiang-Yang Wang et al. [7] proposed a color image retrieval scheme by combining color, texture and shape information. Robustness with respect to the image scale, Illumination and Noise are some of the important aspects to be considered in developing image matching systems. Shao-Hu Peng et al. [11] proposed a visual shape descriptor based on the sectors and shape context of contour lines.

From the discussion so far, it is quite clear that shape is one of the primary visual features in CBIR. Our work focuses on developing a CBIR system based on shape. Shape descriptors are normally classified as contour based and region based. Contour-based shape descriptors primarily use the boundary information 
and neglect the local contents within the shape. But region-based shape descriptors make use of the contents within the shape [20]. Works on CBIR systems based on moment descriptors are in progress [12]. Fuzzy techniques are also being employed in retrieval and recognition [21].

The Precision and Recall (PR) levels of the existing CBIR systems based on Shape have not been very good, when minimal feature sets were used. Extensive feature sets have had a negative impact on the retrieval time. Our work aims at identifying a minimal feature set with high PR levels. A computationally efficient algorithm for getting a first level match is of utmost importance. Computationally expensive refinement can then be used, if required, on this trimmed dataset to get better matches. Considering this situation, we have first used the color and texture properties to extract the objects from the image through segmentation. The shape is extracted from the edges identified using Canny algorithm [13] and from the convex hull containing the prominent edges in the image. A novel similarity measure based on convex hull geometry (Convex Hull Area Ratio - CHAR) is proposed, to retrieve the exact match.

\section{Content-based Image Retrieval Based on Convex Hull Geometry}

Though Convex hull is a good shape representation and description technique, it has not been effectively utilized in CBIR. There has only been very few applications of Convex hull in image retrieval. Lancaster et al [16], used the Convex hull of the brain image in a medical application [17]. The Convex hull derived from the brain's surface was identified as the basis for automating and standardizing global spatial normalization. Their idea was to match the position, orientation and dimensions of the brain to that of a standard atlas brain.

The proposed Content-based Image Retrieval system comprises of the preprocessing steps of image segmentation, smoothing, feature extraction and image retrieval based on the query image [19]. The proposed CBIR is based on the shape signature extracted by employing color based segmentation, edge detection and extraction of the convex hull. When an image is queried, the system determines the shape signature for the image and then computes the similarity measure between the signatures of the query image and the existing database based on the convex hull area ratio (CHAR) comparison and retrieves a specified number of the best matches. The proposed method is detailed below.

In our work, we make use of the K-means clustering algorithm for image segmentation for further processing with $\mathrm{k}=4$. K-means clustering identifies partitions in such a way that objects within each cluster are as close to each other as possible, and as far from objects in other clusters as possible. The number of 
clusters to be partitioned and a distance metric for quantifying the distance between two objects are necessary for K-means clustering. Prior to the application of $\mathrm{K}$-means clustering, the image, which is in the form of a 2D matrix, is rescaled to a $1 \mathrm{D}$ vector. Subsequently, the K- means algorithm is applied, to cluster the image, with k set as 4. After the K- Means Algorithm Is Applied, The 1D Vector Is converted back to $2 \mathrm{D}$ matrix and then the Canny algorithm is used for the detection of edges present in all the clustered sets of the image. The Canny edge detection operator detects a good range of edges in images. The canny algorithm consists of five steps, which are smoothing, finding gradients, non-maximum suppression, double thresholding and edge tracking by hysteresis [13] [14].

Canny edge detection process gives the different edges that are present in the image and then the indices of the shaped content are extracted by employing convex hull method. The convex hull of a set of points is the smallest bounding convex polygon that will contain the set. The convex hull is used for extracting the shape of the image. The approach is that the shape is represented by a single convex hull. The convex hull $H$ of a region is its smallest convex region including it. In order to decrease the effect of noise, we first smooth a boundary prior to partitioning. The representation of the shape is obtained by storing the coordinate values of the convex hull. In a similar manner, the features of the rest of the images in the database are also extracted and stored in a feature library. For preserving scale invariance the convex hull extracted is normalized to unit area by performing a scaling of the co-ordinate system before storing for further analysis. Similarly for preserving translational invariance the co-ordinate system origin is fixed at the centroid of the extracted convex hull.

\subsection{Retrieval Process}

The Image retrieval process takes place after the Image Indexing process. The query image is matched to the images in the DB (database) for image retrieval. Let $Q$ be the query image. We perform the shape extraction for the query image $Q$ and extract the convex hull of the detected shapes. The Q polygon and DB Polygon (of convex hull) are compared by finding ratio of intersected area to union area (CHAR) as explained in Fig. 1. The CHAR values are computed for each image in the DB and stored in an array. The maximum value of this ratio is the closest match. Accordingly, a specified number of images with best match are retrieved from the database. The retrieval process is further explained in detail using Figure 1. 

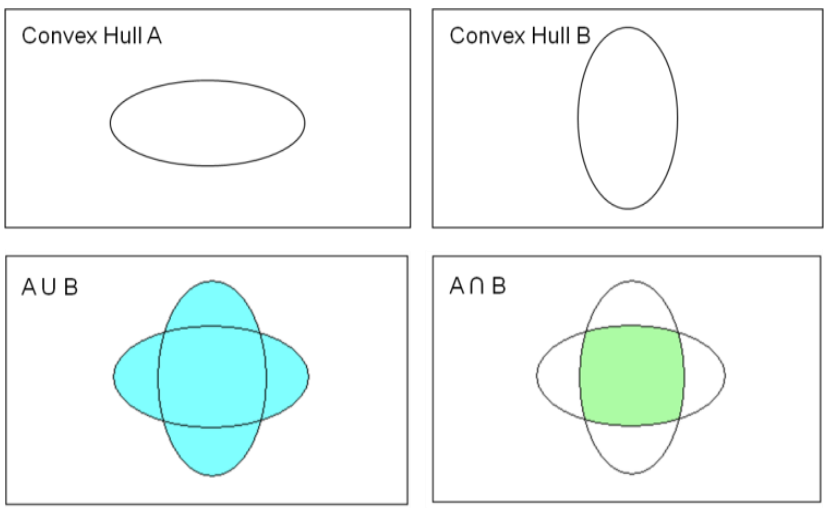

Figure 1

CHAR Computation (i) Convex hull A (ii) Convex Hull B

(iii) Area of union (blue) (iv) Area of intersection (green)

As shown in Figure 1, CHAR is maximum at a value of 1 when the query and database convex hulls are exactly identical. When there is no overlap between the two convex hulls the CHAR value reduces to a minimum value of 0 . The CHAR is a measure of the overlap among the query and retrieved convex hulls.

The convex hull can be obtained by HMT (Hit or Miss Transform) with four structuring elements. Consider A to be a binary image set. Let $B^{i}, i=1,2,3,4$, represent four structuring elements as given below in Figure 2.

$$
B^{1}=\left[\begin{array}{lll}
1 & x & x \\
1 & 0 & x \\
1 & x & x
\end{array}\right], \quad B^{2}=\left[\begin{array}{lll}
1 & 1 & 1 \\
x & 0 & x \\
x & x & x
\end{array}\right], \quad B^{3}=\left[\begin{array}{lll}
x & x & 1 \\
x & 0 & 1 \\
x & x & 1
\end{array}\right], \quad B^{4}=\left[\begin{array}{lll}
x & x & x \\
x & 0 & x \\
1 & 1 & 1
\end{array}\right]
$$

Figure 2

Structuring elements

where the symbol $X$ denotes don't care. $\mathrm{B}^{\mathrm{i}}$ is a structuring element where $\mathrm{i}$ vary from 1 to 4 . These structuring elements are called $\mathrm{B}^{1}, \mathrm{~B} 2, \mathrm{~B}^{3}$ and $\mathrm{B}^{4}$.

Let the initial step be $X_{0}^{i}=A$. The iterative process is conducted as in the $k$ th step

$\mathrm{X}^{\mathrm{i}}{ }_{\mathrm{k}}=\left(\mathrm{X} \circledast \mathrm{B}^{\mathrm{i}}\right) \cup \mathrm{A}, \mathrm{i}=1,2,3,4$ and $\mathrm{k}=1,2,3, \ldots$

If $X_{k}^{i}=X_{k-1}^{i}$, then it converges. That is, in two subsequent iterations, if the output does not change, the algorithm converges.

Let $D^{i}=X_{\text {conv }}^{i}$ 
Finally, the convex hull of $A$ is

$C(A)=\bigcup_{i=1}^{4} D^{i}$

Since there are four different structuring elements, four different point sets are obtained at the end of convergence. The union of these four different point sets gives the convex hull of the given image $\mathrm{A}$.

Similarly, we find the convex hull of the image B. $C(A) \cup C(B)$ and $C(A) \cap C(B)$ are further calculated.

The metric CHAR is given by

$\mathrm{C}(\mathrm{A}) \cap \mathrm{C}(\mathrm{B}) / \mathrm{C}(\mathrm{A}) \cup \mathrm{C}(\mathrm{B})$

\section{Results and Discussion}

This CBIR system has been implemented using MATLAB (version 7.10), has been tested with various query images and appropriate matching images have been recovered from the image database. Test images were taken from the database generated by Wang containing many images stored in the JPEG format [15]. When an image is queried, the system establishes a shape feature for the image and then computes the similarity measure between the shape feature of the query image and the shape features of all images existing in the database, so that, a specified number of database images similar to the query image are retrieved.

To retrieve the image from the image database, the query image is preprocessed to normalize the intensity levels of the input image. The output objects extracted from the input query image after the preprocessing stage is given in Row 2 of Figure 3. To extract the shape feature from the image, initially, the image in RGB color space is converted to gray scale image. Since, the mean filter can act on only one color channel. The mean filter is especially useful for reducing speckle noise and salt and pepper noise. The image is converted into gray scale using Craig's formula. After converting the RGB image into gray scale image, K-means clustering algorithm $(\mathrm{k}=4)$ is used for the image segmentation for the further process. Before applying the K-means clustering, the image, which is in the form of $2 \mathrm{D}$ vector, is rescaled to a $1 \mathrm{D}$. In this $\mathrm{K}$ means clustering process the image pixels are grouped under the color. The output image of the clustering process is shown in the Row 3 of Figure 3. After the clustering process using K-Means algorithm, the $1 \mathrm{D}$ image is again converted into $2 \mathrm{D}$ image format. Then canny algorithm is used for the detection of different edges present in all the clustered 
sets of the image. The canny algorithm comprises of five steps, they are smoothing, finding gradients, non-maximum suppression, double thresholding and edge tracking by hysteresis. The image after detecting the edge is shown in the Row 4 of Figure 3.

After applying canny algorithm to determine the edges of the segmented image, the edges are tracked and then smoothed to remove the number of connected components. Then we get the prominent shapes that are present in the image and then the $\mathrm{x}$, $\mathrm{y}$ values of the extracted convex hull polygon of the detected edges are stored.
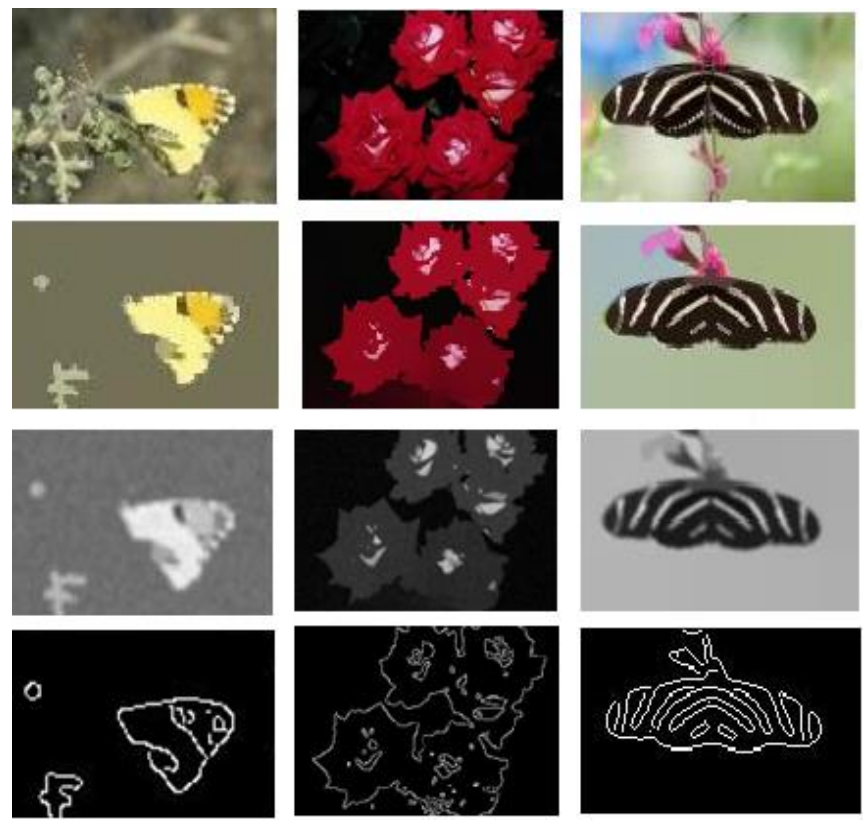

Figure 3

Row 1 - Original images, Row 2 - Objects extracted from the original images, Row 3 - Clustered gray scale images, Row 4 - Edges detected by Canny algorithm

Retrieval is performed based on the proposed Convex Hull Area Ratio (CHAR) method, as explained by Figure 1 and Figure 4. 


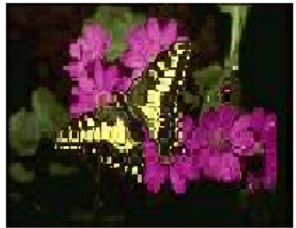

(a) Query image

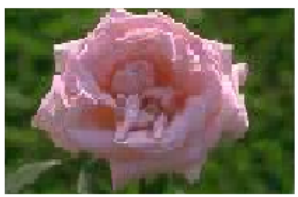

(c) A matched image from the database

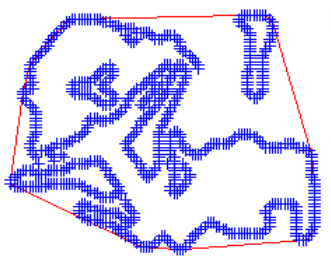

(b) Convex hull containing the prominer edges of the query image

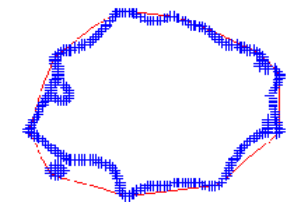

(d) Convex hull contrining the prominent edges of the matched image

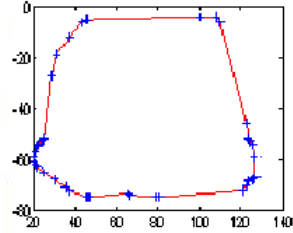

(e) Union of the Convex hulls of the query and $m$ atched images

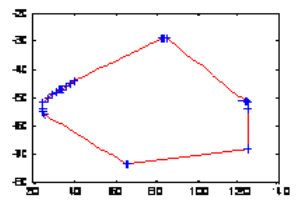
(f) Intersection of the Convex hulls of
the query and $m$ atched im ages

\section{Figure 4}

Union and Intersection areas of the query image and a matched image. Ratio of Intersection to Union will be close to 1 for a good match and low for dissimilar ones.

During the computation of the convex hull, the scale invariance is preserved by normalizing the area of each convex hull to unity. This assures that only the relative spread of the shape features are compared and similar shapes give a better CHAR. Translational invariance is preserved by setting the origin to the centroid of the convex hull polygon.

Retrieved images that correspond to the input query image are shown in Figure 5 and Figure 6.
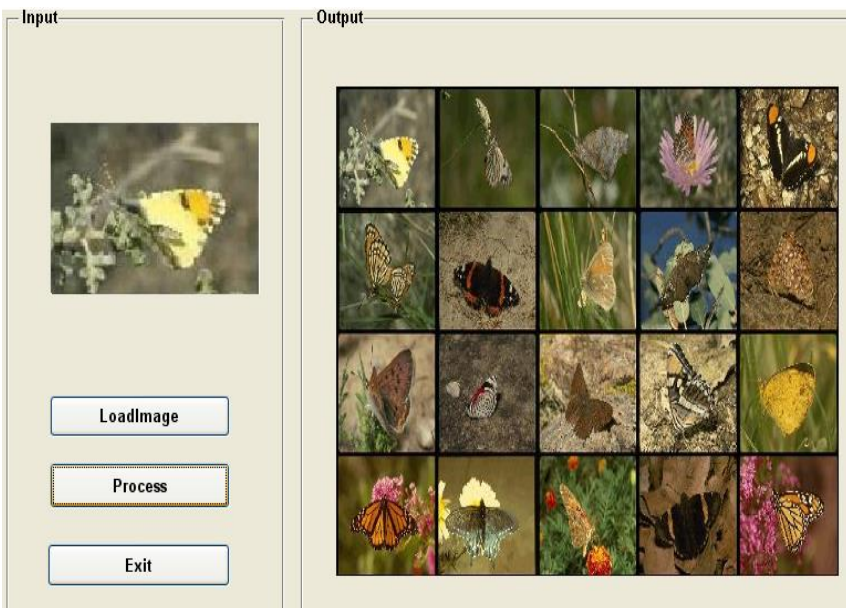

Figure 5

Sample Output - Query Image and Retrieved images 


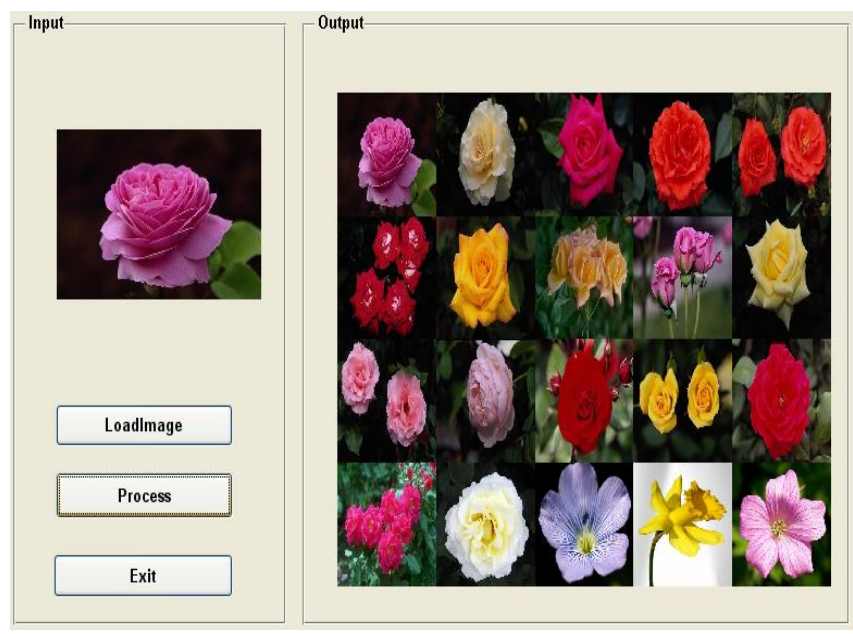

Figure 6

Sample Output - Query Image and Retrieved images

\subsection{Comparative Analysis}

The performance can be identified by using precision and recall. Precision is the fraction of retrieved images that are relevant to the query image, while recall is the fraction of relevant images that are retrieved from the database. Precision indicates the accuracy and Recall indicates the relevance of retrieval.

precision $=\frac{\text { Count of retrieved images relevant to the query image }}{\text { Total count of images retrieved }}$

recall $=\frac{\text { Count of retrieved images relevant to the query image }}{\text { Total count of relevant images in the database }}$

Using Equation (4) and Equation (5), the precision and recall values for the query image are calculated for the proposed method. The values obtained from the calculation are given in Table 1 . The precision values are also compared with some of the existing methods and plotted in Figure 7. 


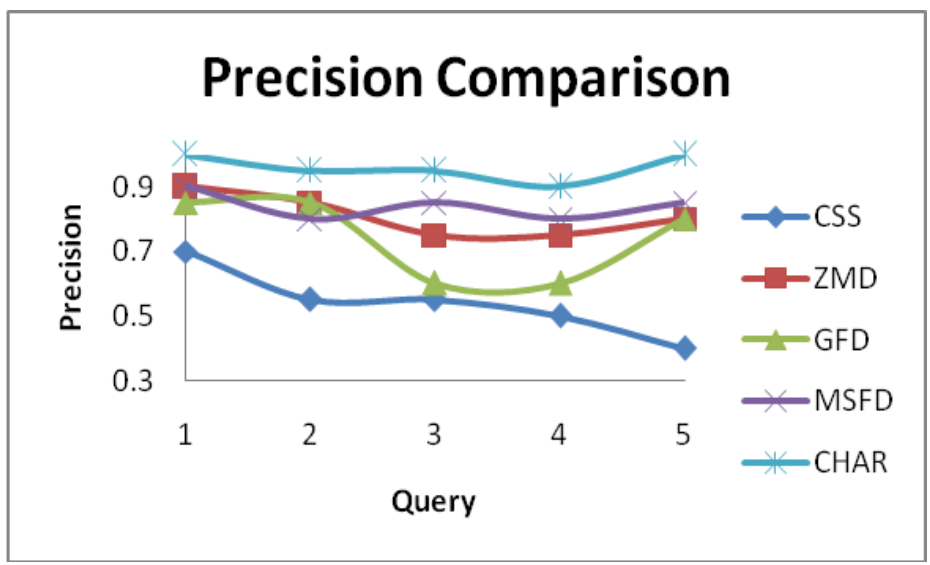

Figure 7

Comparison of Precision

Table 1

Precision (P) and Recall (R) Statistics for the proposed CHAR (Convex Hull Area Ratio) Method on the best 15, 20 and 40 retrieved images

\begin{tabular}{|c|c|c|c|c|c|c|}
\hline \multirow[t]{2}{*}{ Query } & \multicolumn{2}{|c|}{$\mathrm{N}=15$} & \multicolumn{2}{|c|}{$\mathrm{N}=20$} & \multicolumn{2}{|c|}{$\mathrm{N}=40$} \\
\hline & $\mathrm{P}$ & $\mathrm{R}$ & $\mathrm{P}$ & $\mathrm{R}$ & $\mathrm{P}$ & $\mathrm{R}$ \\
\hline 1 & 1 & 0.30 & 1 & 0.40 & 0.9 & 0.72 \\
\hline 2 & 1 & 0.42 & 1 & 0.58 & 0.65 & 0.74 \\
\hline 3 & 1 & 0.30 & 0.95 & 0.38 & 0.98 & 0.78 \\
\hline 4 & 1 & 0.30 & 1 & 0.40 & 0.9 & 0.72 \\
\hline 5 & 1 & 0.30 & 1 & 0.40 & 0.9 & 0.72 \\
\hline 6 & 0.93 & 0.28 & 1 & 0.40 & 0.93 & 0.74 \\
\hline 7 & 0.93 & 0.28 & 0.9 & 0.36 & 0.88 & 0.70 \\
\hline 8 & 1 & 0.30 & 0.95 & 0.38 & 0.88 & 0.70 \\
\hline 9 & 0.93 & 0.28 & 0.9 & 0.36 & 0.8 & 0.64 \\
\hline 10 & 0.86 & 0.26 & 0.9 & 0.36 & 0.83 & 0.66 \\
\hline
\end{tabular}


Figure 7 shows the graph for the comparative analysis between the proposed Convex Hull Area Ratio (CHAR) method and existing methods like CSS (Curvature Scale Space), ZMD (Zernike Moment Descriptor), GFD (Geometric Fourier Descriptor) and MSFD (Multiple Shape Feature Descriptor) on 5 queries for a set of best 20 images retrieved. The data for other methods is from [4] [7].

Based on Figure 7, the proposed method has effectively retrieved the images with high precision, in comparison with other existing methods. The retrieval is also quite fast since only the convex hull points are used in the matching process. The Precision - Recall table and the Precision comparison establish the superiority of the proposed CHAR-based Content-based Image Retrieval System. This work can further be improved by preserving rotation invariance. Feature set can also be extended to include inner shape features and texture dimensions by grouping the edge sets based on their color and texture properties. However this will increase the complexity of the CBIR system and can be justified only when the additional computation is mandated for specific applications to delineate color as well as texture.

\section{Conclusions}

In this paper, we have proposed a novel and efficient CBIR system based on Shape Signature to retrieve relevant images from image database for a given query image. In this method, when an image is queried, the system establishes shape feature for the image and then the ratio of the intersected area to union area of the convex hull polygons of the query and database images are found and stored in an array. Subsequently, similarity measure is performed and the maximum value of the ratio indicates the closest match. Here we first pre-process the image by using K-means clustering algorithm for image segmentation based on color. Edges are extracted using the Canny algorithm. We have proposed and implemented the CHAR method for efficient matching and retrieval. The implementation results illustrate that this novel image retrieval process effectively retrieves the images that are close to the query image from the database.

\section{References}

[1] Ricardo da S. Torresa, Alexander X. Falcaoa, Marcos A. Gonçalvesb, Joao P. Papaa, Baoping Zhang, Weiguo Fanc and Edward A. Foxc: A Genetic Programming Framework for Content-based Image Retrieval, Journal of Pattern Recognition, Vol. 42, No. 2, 2009, pp. 283-292

[2] Ying Liu, Dengsheng Zhang, Guojun Lu and Wei-Ying Ma: A Survey of Content-based Image Retrieval with High-level Semantics, Journal of Pattern Recognition, Vol. 40, No. 1, 2007, pp. 262-282

[3] Amit Jain, Ramanathan Muthuganapathy and Karthik Ramani: Contentbased Image Retrieval Using Shape and Depth from an Engineering Database, In Proceedings of the Third International Conference on Advances in Visual Computing, 2007, pp. 255-264 
[4] Dengsheng Zhang and Guojun Lu: A Comparative Study of Curvature Scale Space and Fourier Descriptors for Shape-based Image Retrieval, Journal of Visual Communication and Image Representation, Vol. 14, No. 1, 2003, pp. 39-57

[5] Dengsheng Zhang and Guojun Lu: Shape-based Image Retrieval using Generic Fourier Descriptor, Journal of Signal Processing: Image Communication, Vol. 17, No. 10, 2002, pp. 825-848

[6] Chia-Hung Wei, Yue Li, Wing Yin Chau and Chang-Tsun Li: Trademark Image Retrieval Using Synthetic Features for Describing Global Shape and Interior Structure, Journal of Pattern Recognition, Vol. 42, No. 3, 2009, pp. 386-394

[7] Xiang-Yang Wang, Yong-Jian Yu, and Hong-Ying Yang: An Effective Image Retrieval Scheme using Color, Texture and Shape Features, Journal of Computer Standards \& Interfaces, Vol. 33, 2010, pp. 59-68

[8] Chuan-Cheng Wang and Ling-Hwei Chen: Content-based Color Trademark Retrieval System Using Hit Statistic, International Journal of Pattern Recognition and Artificial Intelligence, Vol. 16, No. 5, 2002, pp. 603-619

[9] Ying Liu, Dengsheng Zhang, Guojun Lu and Wei-Ying Ma: A Survey of Content-based Image Retrieval with High-level Semantics, Journal of Pattern Recognition, Vol. 40, No. 1, 2007, pp. 262-282

[10] H. B. Kekre and Dhirendra Mishra: Four Walsh Transform Sectors Feature Vectors for Image Retrieval from Image Databases, International Journal of Computer Science and Information Technologies, Vol. 1, No. 2, 2010, pp 33-37

[11] Shao-Hu Peng, Deok-Hwan Kim, Seok-Lyong Lee and Chin-Wan Chung: A Visual Shape Descriptor Using Sectors and Shape Context of Contour Lines, Journal of Information Sciences, Vol. 180, No. 16, 2010, pp. 29252939

[12] Srinivasa Rao, S. Srinivas Kumar and B.Chandra Mohan: Content-based Image Retrieval Using Exact Lengendre Moments And Support Vector Machine, The International Journal of Multimedia \& Its Applications, Vol. 2, No. 2, 2010, pp. 69- 79

[13] Ravichandran and Ananthi: Color Skin Segmentation Using K-Means Cluster, International Journal of Computational and Applied Mathematics, Vol. 4, No. 2, 2009, pp. 153-157

[14] Krishnan N, Justin Varghese, S. Saudia, Santhosh. P.Mathew et al: A New Adaptive Class of Filter Operators for Salt and Pepper Impulse Corrupted Images, International Journal of Imaging Science and engineering (IJISE), Vol. 1, No. 2, 2007, pp. 44-51 
[15] Ritendra Datta, Dhiraj Joshi, Jia Li, and James Z. Wang: Image Retrieval: Ideas, Influences, and Trends of the New Age, ACM Computing Surveys, Vol. 40, No. 2, 2008, Article 5

[16] Lancaster, JL, Fox, PT, Downs, H, Nickerson, DS, Hander, TA, Mallah, ME, Kochunov, PV and Zamarripa, F: Global Spatial Normalization of Human Brain Using Convex Hulls, The Journal of Nuclear Medicine, Vol. 40, No. 6, 1999, pp. 942-955

[17] Szabolcs Sergyán: A New Approach of Face Detection-based Classification of Image Databases, Acta Polytechnica Hungarica, Journal of Applied Sciences, Vol. 6, No. 1, 2009, pp. 175-184

[18] S. P. Mathew and P. Samuel: A Novel Image Retrieval System using an Effective Region-based Shape Representation Technique, International Journal of Image Processing (IJIP), Vol. 4, No. 5, Dec. 2010, pp. 509-517

[19] Santhosh P. Mathew, Valentina E. Balas, Zachariah K. P, Philip Samuel: A Content-based Image Retrieval System Based on Polar Raster Edge Sampling Signature, Acta Polytechnica Hungarica, Journal of Applied Sciences, Vol. 11, No. 3, 2014, pp. 25-36

[20] Loris Nanni, Alessandra Lumini, Sheryl Brahnam: Ensemble of Shape Descriptors for Shape Retrieval and Classification, International Journal of Advanced Intelligence Paradigms (IJAIP), Vol. 6, No. 2, 2014, pp. 136-156

[21] S. Bharathi, R. Sudhakar, Valentina E. Balas : Biometric Recognition using Fuzzy Score Level Fusion, International Journal of Advanced Intelligence Paradigms (IJAIP), Vol. 6, No. 2, 2014, pp. 81-94 